\title{
Upregulated miR-200c is associated with downregulation of the functional receptor for severe acute respiratory syndrome coronavirus 2 ACE2 in individuals with obesity
}

\author{
Jayanthi Bellae Papannarao ${ }^{1}$, Daryl O. Schwenke ${ }^{1}$, Patrick Manning ${ }^{2}$ and Rajesh Katare $\mathbb{I D}^{1 凶}$ \\ (C) The Author(s), under exclusive licence to Springer Nature Limited 2021
}

\begin{abstract}
Obesity is a risk factor for coronavirus disease 2019 (COVID-19) infection, with studies demonstrating the prevalence of individuals with obesity admitted with COVID-19 ranging between 30 and 60\%. We determined whether early changes in microRNAs (miRNAs) are associated with dysregulation of angiotensin-converting enzyme 2 (ACE2), the specific functional receptor for severe acute respiratory syndrome coronavirus 2. ACE2 is a membrane-bound enzyme that catalyzes the conversion of angiotensin II to angiotensin 1-7 the latter having cardioprotective and vasorelaxation effects. Quantitative real-time PCR analysis of plasma samples for circulating miRNAs showed upregulation of miR-200c and miR-let-7b in otherwise healthy individuals with obesity. This was associated with significant downregulation of ACE2, a direct target for both miRNAs, in individuals with obesity. Correlation analysis confirmed a significant negative correlation between ACE2 and both the miRNAs. Studies showed that despite being the functional receptor, inhibition/downregulation of ACE2 did not reduce the severity of COVID-19 infection. In contrast, increased angiotensin II following inhibition of ACE2 may increase the severity of the disease. Taken together, our novel results identify that upregulation of miR-200c may increase the susceptibility of individuals with obesity to COVID-19. Considering miRNA are the earliest molecular regulators, the level of circulating miR-200c could be a potential biomarker in the early identification of those at the risk of severe COVID-19.
\end{abstract}

International Journal of Obesity (2022) 46:238-241; https://doi.org/10.1038/s41366-021-00984-2

\section{INTRODUCTION}

Obesity is considered an epidemic of the 21 st century, with recent analysis suggesting that obesity prevalence could reach $20 \%$ of the world's population by 2025 [1]. Obesity is associated with the development of type 2 diabetes mellitus, cardiovascular diseases including stroke and hypertension, certain types of cancers, and several other pathological conditions $[2,3]$. Severe acute respiratory syndrome coronavirus 2 (SARS-CoV-2) causing coronavirus disease 2019 (COVID-19) pandemic has a devastating and chronic effect on the health of an individual, killing more than 2.6 million people (to date) worldwide. Dissemination of a vaccine and immunization program against COVID-19 is likely to reduce the intensity and mortality rate in most cohorts of patients without any other risk factors. However, people who fall within a high-risk category, such as obesity, remain susceptible to ongoing complications associated with COVID-19. Obesity is considered a risk factor for COVID-19 infection, with studies demonstrating the prevalence of individuals with obesity admitted with COVID-19 ranging between 30 and 60\% [4]. Once infected, individuals with obesity develop a serious illness associated with a higher mortality rate [4]. Excessive adiposity is thought to be causally linked to this high mortality with COVID-19 infection [5] because increased fat disrupts normal metabolism, activates multiple inflammatory cytokines and reduces anti-inflammatory agents such as adiponectin, all of which normally protect the lung against infection. Notably, the exact molecular mechanism(s) remain elusive yet are essential for furthering our understanding and establishing preventative strategies against the insult of COVID-19.

MicroRNAs (miRNAs) are small non-coding RNA molecules that constitute $1-5 \%$ of the human genome and regulate at least $30 \%$ of all protein-coding genes [6]. Accumulating evidence from the past decade has identified miRNAs as critical elements in several physiological and pathological processes [6]. Further, miRNAs are secreted into the circulation, where they remain stable, hence serving as a valuable diagnostic and prognostic biomarker of diseases [6-8]. Our recent study identified dysregulation of circulating miRNAs associated with diabetes, insulin signaling, cell survival, and cardiovascular diseases in individuals with obesity [9]. Interestingly, the majority of the miRNAs were normalized following acute weight loss in these individuals, demonstrating

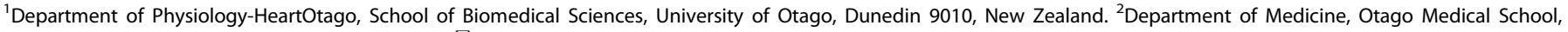
University of Otago, Dunedin 9010, New Zealand. ${ }^{\circledR}$ email: rajesh.katare@otago.ac.nz 
the role of miRNAs as biomarker [9]. In support of this, other studies have demonstrated the diagnostic potential of miRNAs in cardiovascular diseases, diabetes, and cancer [6-8].

In this study, we aimed to determine whether obesity-induced changes in miRNAs is associated with dysregulation of angiotensin-converting enzyme 2 (ACE2), the specific functional receptor for SARS-CoV-2 [10]. ACE2 is a membrane-bound enzyme that catalyzes the conversion of angiotensin II to angiotensin 1-7, and previous studies suggested that ACE2 is a key enzyme for the balance between pro-inflammatory effects of angiotensin (Ang) II and anti-inflammatory effects of Ang 1-7 [11]. This can lay the foundation for future studies to determine if dysregulation of ACE2 could be the underlying mechanism for increasing the risk for developing COVID-19 infection in individuals with obesity. In particular, we tested two miRNAs, miR-200c and miR-let-7b, both of which directly target ACE2.

\section{SUBJECTS AND METHODS}

\section{Participants and sample collection}

The Health and Disability Ethics Committee of New Zealand approved the use of samples for this study. Participants were identified from the ongoing Prevention Of WEight Regain (POWER) study (Clinical Trials Registry number: ACTRN12612000069853), which was designed to assess the effects of cabergoline on the prevention of weight regain in individuals with obesity. All participants in the POWER study provided plasma samples at baseline [9, 12]. Informed consent was obtained from each patient for the collection and use of samples in this study, and the study protocol conforms to the ethical guidelines of the 1975 Declaration of Helsinki as reflected in a priori approval by the New Zealand Health and Disability Ethics Committee. Samples collected from 31 obese female participants for the present study were randomly selected from the 221 obese female individuals recruited in the POWER study. Men were excluded as there were very few recruited into the POWER study. Plasma samples also collected from 30 age- $( \pm 1.5$ years $)$ matched female lean individuals recruited through public advertising. Exclusion criteria for lean and obese participants were pregnancy, diabetes, malignancy, cardiac disease, other vascular diseases, uncontrolled hypertension, psychiatric disease, or any other significant medical condition.

\section{RNA extraction and quantitative real-time PCR analysis}

Total RNA was extracted from plasma using QIAgen miRNAeasy mini kit following the manufacturer's instruction and described in our earlier studies [6-8]. Total RNA was reverse transcribed followed by amplification using miR-200c and let $7 \mathrm{~b}$ and internal controls miR-16 and miR-24 specific stem-loop structure and reverse transcription primers (all from Thermofisher Scientific). miRNA expression was normalized to internal controls (both miR-16 and miR-24a expression were comparable between lean and obese participants) and presented as DCT $\left(2^{-\Delta C t}\right)$ expression. All the experiments were repeated at least two independent times, and the data from each independent repeat were averaged.

\section{ELISA for circulating ACE2 and angiotensin II}

Plasma $(50 \mu \mathrm{L})$ from obese and lean individuals was used to measure the level of ACE2 using the commercial kit (Human ACE-2 DuoSet ELISA, R\&D Systems) and angiotensin II by Sandwich Elisa using primary antibody against angiotensin II (Thermo fisher, NZ), following the manufacturer's instructions.

\section{Statistical analysis}

All statistical analyses were performed using GraphPad Prism (version 8). Shapiro-Wilk test was used to test the normality. A non-parametric Mann-Whitney $U$ test was used to analyze the quantitative real-time PCR analysis and ELISA results. Data are expressed as the mean \pm standard error of the mean (SEM). Spearman correlation analysis was used to determine the correlation between ACE2 and miRNAs. A $p$ value $<0.05$ was considered statistically significant.

\section{RESULTS}

Baseline clinical data showed significantly higher BMI and blood pressure in individuals with obesity (Supplemental Table 1).
Quantitative real-time PCR analysis of the total RNA isolated from plasma showed a significant upregulation of circulating miR-200c in individuals with obesity compared to the lean individuals $(P<$ 0.0001 vs. lean, Fig. $1 \mathrm{~A})$. While miR-let-7b expression was higher in individuals with obesity, it was not statistically $(P=0.312$, Fig. 1B). ELISA revealed a significant decrease in the level of secreted ACE2, the direct target of miR-200c and miR-let-7b in individuals with obesity ( $P \leq 0.0001$, Fig. $1 C)$. Circulating angiotensin II levels were marginally increased in individuals with obesity, although this was not statistically significant compared to lean individuals (Supplementary Fig. 1). While this was contrary to our expectation, this is possibly due to fewer sample numbers or the absence of other associated comorbidities. To our knowledge, there are no clinical studies that have measured the level of circulating angiotensin II in individuals with obesity, except one showing increased circulating angiotensin II in patients with obesity with cardiovascular disease and type 2 diabetes [13].

Next, to determine the correlation between miR-200c upregulation and reduced levels of ACE2, we used the Spearman correlation coefficient, which showed a significant negative correlation between miR-200c and ACE2 irrespective of the group $(r=-0.3054, P=0.0247$, Fig. 2B). Interestingly, although miR-let$7 \mathrm{~b}$ expression was not significantly different in individuals with obesity, correlation analysis showed a significant negative correlation between miR-let-7b and ACE2 $(r=-0.3061, P=$ 0.0258, Fig. 2C).

\section{DISCUSSION}

A crucial finding from our study is that upregulation of miR-200c associated with obesity could be a molecular mechanism increasing the risk in individuals. miR-200c is located in the intergenic region of chromosome 12, along with miR-141, and is highly expressed in epithelial cells, including type 2 alveolar epithelial cells [14]. In the fetal lung, upregulation of miR-200c contributed to the differentiation of type II alveolar epithelial cells [14]. On the other hand, a higher miR-200c expression was associated with shorter survival in patients with non-small cell lung cancer [15]. Further, miR-200c directly targets ACE2, and our results confirmed a strong negative correlation between miR-200c and ACE2. Interestingly, miR-let-7b, another known modulator of ACE2, did not reach significance in individuals with obesity, which is likely due to increased variability in miR-let-7b expression among study participants. However, it showed a significant negative correlation to ACE2.

Being the main receptor for SARS-CoV-2, ACE2 is considered a promising therapeutic target for the treatment of COVID-19 [16]. Therefore, it is intriguing to consider that increased miR-200c may benefit the individuals with obesity by directly targeting ACE2, which is supported by our results. However, obesity is strongly and independently associated with adverse outcomes of COVID-19 [5]. ACE2 is expressed in nearly all human organs in varying degrees and plays a crucial role in cellular homeostasis. In the lungs, like miR-200c, ACE2 is mainly expressed on type II alveolar epithelial cells. It is an important component of the renin-angiotensin system signaling throughout the body and exhibits anti-inflammatory, anti-remodeling, and anti-proliferative properties by reducing angiotensin II levels. Importantly, a previous study has shown a proportional increase in ACE2 activity in both circulation and different tissues, including lungs, heart, and pancreas, in a mouse model of diabetes [17]. It is important to note that COVID19 patients using angiotensin-converting enzyme inhibitors or angiotensin receptor blockers, which upregulate ACE2 levels, do not show higher mortality rates or increased disease severity [18]. Therefore, having a low level of ACE2 is unlikely to be beneficial. On the other hand, when individuals with the low level of ACE2, such as the individuals with obesity in our study, is affected with COVID-19, binding of SARS-CoV-2 to ACE2 is likely to lead to 
A

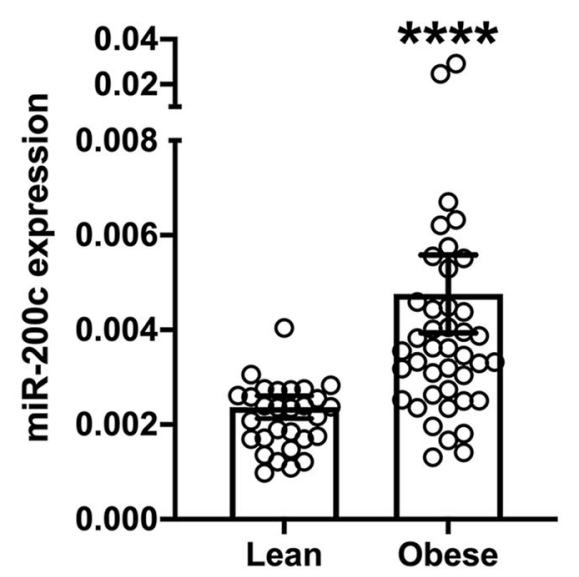

C

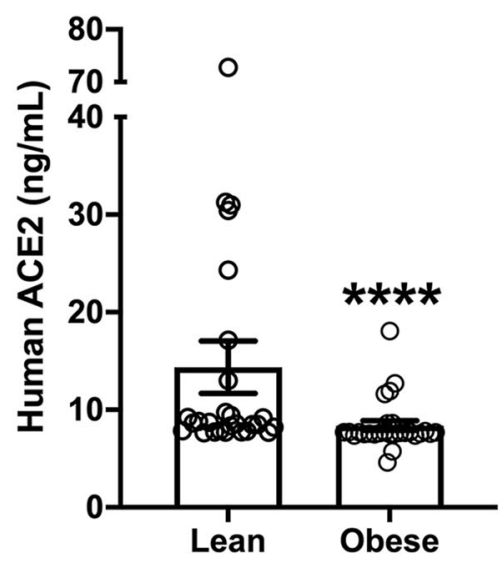

B

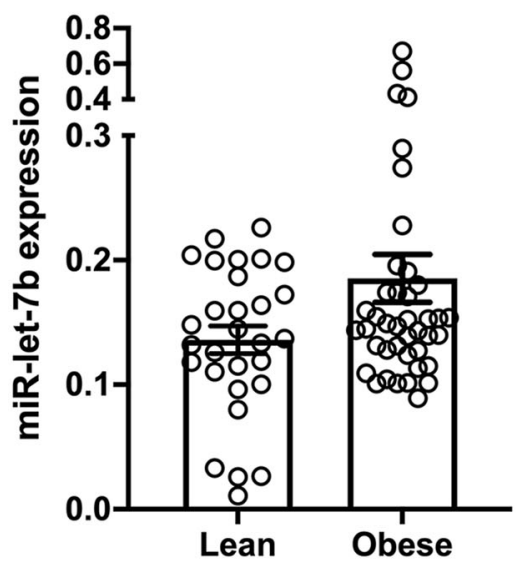

Fig. 1 Upregulation of miR-200c in individuals with obesity. Quantitative scatter plot bar graphs showing the RT-PCR analysis of miR-200c (A) and let-7b (B) in the study participants. All the experiments were repeated at least two independent times. C Quantitative scatter plot bar graph showing human ACE2 levels in study participants measured by ELISA. ELISA was performed in triplicates. The data are presented as mean \pm SEM. $N=30$ in lean and 31 in individuals with obesity. ${ }^{* * *} P<0.0001$ vs. lean participants.

A

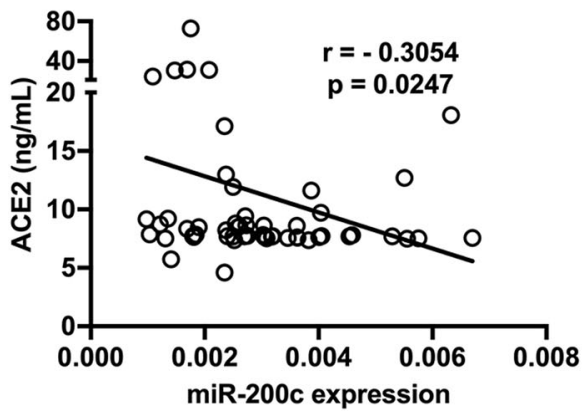

B

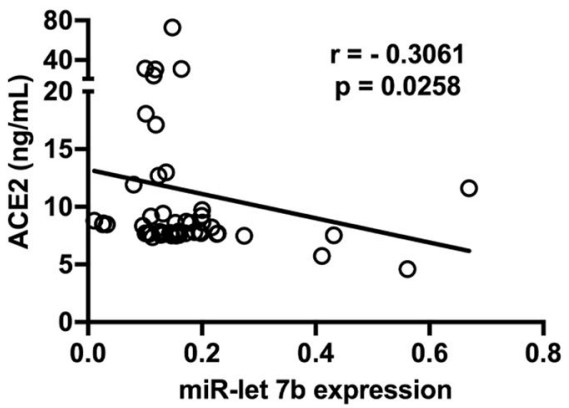

Fig. 2 Negative correlation between miR-200c and ACE2. Line graphs with scatter plots of Spearman correlation analysis showing a significant negative correlation between ACE2 and miR-200c (A) and let-7b (B).

reduced ACE2 cell surface expression, resulting in critically low ACE2 levels and exaggerated angiotensin II signaling, leading to severe form of the disease [19].

While it can be argued that measurement of ACE2 level is sufficient to identify those at the risk of developing severe disease, dysfunction in miRNAs always precede changes in protein and cellular structure/function and, thus, are the earliest molecular regulators. We, along with others, have demonstrated the changes in miRNA well before the changes are identified at the target protein level [6-8]. Therefore, the early detection of increased miR200c expression in individuals with obesity from a simple blood test could significantly mitigate the risk of contracting COVID-19. 
In support of this, previous studies have suggested that measuring the upstream regulators of ACE2, including miRNAs [20] or the circulating level of ACE2 [21] could predict the outcome in patients affected with COVID-19. While our study has a limited number of participants, this is the first proof of concept study showing the correlation and association between miR-200c and ACE2, the functional receptor of the SARS-CoV2 virus. Therefore, further studies with a higher number of participants will be required to validate the use of circulating miR-200c as a biomarker of COVID-19 in individuals with obesity. Moreover, it will be interesting to determine the level of miR-200c in individuals affected with COVID-19 to establish its role as a biomarker to identify those at risk of contracting COVID-19.

One limitation of our study is that all the participants were women. Studies published during the earlier stages of COVID-19 pandemic $[22,23]$ demonstrated higher mortality in men than women. However, recent studies published earlier this year suggest that the mortality rate due to COVID-19 is higher in women with obesity $[24,25]$. The difference in findings could be due to an increase in the incidence of COVID, allowing to include more sample size. Nevertheless, these data further strengthens our study's aim that identifying women with obesity at risk of contracting COVID-19 will be highly beneficial.

\section{REFERENCES}

1. Collaboration NCDRF. Trends in adult body-mass index in 200 countries from 1975 to 2014: a pooled analysis of 1698 population-based measurement studies with 19.2 million participants. Lancet. 2016;387:1377-96.

2. Williams EP, Mesidor M, Winters K, Dubbert PM, Wyatt SB. Overweight and obesity: prevalence, consequences, and causes of a growing public health problem. Curr Obes Rep. 2015;4:363-70.

3. Guh DP, Zhang W, Bansback N, Amarsi Z, Birmingham CL, Anis AH. The incidence of co-morbidities related to obesity and overweight: a systematic review and meta-analysis. BMC Public Health. 2009;9:88.

4. Helvaci N, Eyupoglu ND, Karabulut E, Yildiz BO. Prevalence of obesity and its impact on outcome in patients with COVID-19: a systematic review and metaanalysis. Front Endocrinol. 2021;12:598249.

5. Lockhart SM, O'Rahilly S. When two pandemics meet: why is obesity associated with increased COVID-19 mortality? Med. 2020;1:33-42.

6. Rawal S, Manning P, Katare R. Cardiovascular microRNAs: as modulators and diagnostic biomarkers of diabetic heart disease. Cardiovasc Diabetol. 2014;13:44.

7. Lew JK, Pearson JT, Saw E, Tsuchimochi H, Wei M, Ghosh N, et al. Exercise regulates microRNAs to preserve coronary and cardiac function in the diabetic heart. Circ Res. 2020;127:1384-1400.

8. Fomison-Nurse I, Saw EEL, Gandhi S, Munasinghe PE, Van Hout I, Williams MJA, et al. Diabetes induces the activation of pro-ageing miR-34a in the heart, but has differential effects on cardiomyocytes and cardiac progenitor cells. Cell Death Differ. 2018;25:1336-49.

9. Manning P, Munasinghe PE, Bellae Papannarao J, Gray AR, Sutherland W, Katare R. Acute weight loss restores dysregulated circulating microRNAs in individuals who are obese. J Clin Endocrinol Metab. 2019;104:1239-48.

10. Li W, Moore MJ, Vasilieva N, Sui J, Wong SK, Berne MA, et al. Angiotensinconverting enzyme 2 is a functional receptor for the SARS coronavirus. Nature. 2003;426:450-4.

11. Sarzani R, Giulietti F, Di Pentima C, Giordano P, Spannella F. Disequilibrium between the classic renin-angiotensin system and its opposing arm in SARS-CoV2-related lung injury. Am J Physiol Lung Cell Mol Physiol. 2020;319:L325-L336.

12. Manning PJ, Grattan D, Merriman T, Manning T, Williams S, Sutherland W. Pharmaceutical interventions for weight-loss maintenance: no effect from cabergoline. Int J Obes. 2018;42:1871-9.

13. Al-Daghri NM, Bindahman LS, Al-Attas OS, Saleem TH, Alokail MS, Alkharfy KM, et al. Increased circulating ANG II and TNF-alpha represents important risk factors in obese saudi adults with hypertension irrespective of diabetic status and BMI. PLoS ONE. 2012;7:e51255.

14. Benlhabib $H$, Guo W, Pierce BM, Mendelson CR. The miR-200 family and its targets regulate type II cell differentiation in human fetal lung. J Biol Chem. $2015 ; 290: 22409-22$
15. Tejero R, Navarro A, Campayo M, Vinolas N, Marrades RM, Cordeiro A, et al. miR141 and miR-200c as markers of overall survival in early stage non-small cell lung cancer adenocarcinoma. PLoS ONE. 2014;9:e101899.

16. Lu D, Chatterjee S, Xiao K, Riedel I, Wang Y, Foo R, et al. MicroRNAs targeting the SARS-CoV-2 entry receptor ACE2 in cardiomyocytes. J Mol Cell Cardiol. 2020;148:46-9.

17. Roca-Ho H, Riera M, Palau V, Pascual J, Soler MJ. Characterization of ACE and ACE2 expression within different organs of the NOD mouse. Int J Mol Sci. 2017;18 (3):563

18. Fosbol EL, Butt JH, Ostergaard L, Andersson C, Selmer C, Kragholm K, et al. Association of angiotensin-converting enzyme inhibitor or angiotensin receptor blocker use with COVID-19 diagnosis and mortality. JAMA. 2020;324:168-77.

19. AlGhatrif M, Cingolani O, Lakatta EG. The dilemma of coronavirus disease 2019, aging, and cardiovascular disease: insights from cardiovascular aging science. JAMA Cardiol. 2020;5:747-8.

20. Elemam NM, Hasswan $H$, Aljaibeji $H$, Sulaiman N. Circulating soluble ACE2 and upstream microRNA expressions in serum of type 2 diabetes mellitus patients. Int J Mol Sci. 2021;22:10.

21. Kragstrup TW, Singh HS, Grundberg I, Nielsen AL, Rivellese F, Mehta A, et al. Plasma ACE2 predicts outcome of COVID-19 in hospitalized patients. PLoS ONE. 2021;16:e0252799.

22. Palaiodimos L, Kokkinidis DG, Li W, Karamanis D, Ognibene J, Arora S, et al. Severe obesity, increasing age and male sex are independently associated with worse inhospital outcomes, and higher in-hospital mortality, in a cohort of patients with COVID-19 in the Bronx, New York. Metabolism. 2020;108:154262.

23. Williamson EJ, Walker AJ, Bhaskaran $\mathrm{K}$, Bacon $\mathrm{S}$, Bates $\mathrm{C}$, Morton $\mathrm{CE}$, et al. Factors associated with COVID-19-related death using OpenSAFELY. Nature. 2020;584:430-6.

24. Naaraayan A, Nimkar A, Pant S, Hasan A, Durdevic M, Elenius $H$, et al. Sex disparity in the effect of obesity in hospitalized COVID-19 patients: a retrospective cohort study from the New York City metropolitan area. Cureus. 2021;13:e15235.

25. Peters SAE, MacMahon S, Woodward M. Obesity as a risk factor for COVID-19 mortality in women and men in the UK biobank: Comparisons with influenza/ pneumonia and coronary heart disease. Diabetes Obes Metab. 2021;23:258-62.

\section{ACKNOWLEDGEMENTS}

This study was supported by funding from Lottery Health Research Funding (R-LHR2020-128280), the University of Otago Research Funding and the Department of Physiology funding.

\section{AUTHOR CONTRIBUTIONS}

JBP processed plasma samples, conducted all the experiments, analyzed data, interpreted results and wrote the first draft of the manuscript. DOS co-designed the study and provided feedback on the manuscript. PM supervised recruitment of study participants, collection of plasma samples and provided feedback on the manuscript. RK designed the study, trained JBP with experimental techniques, supervised data analysis and wrote the final draft of the manuscript.

\section{COMPETING INTERESTS}

The authors declare no competing interests.

\section{ADDITIONAL INFORMATION}

Supplementary information The online version contains supplementary material available at https://doi.org/10.1038/s41366-021-00984-2.

Correspondence and requests for materials should be addressed to Rajesh Katare.

Reprints and permission information is available at http://www.nature.com/ reprints

Publisher's note Springer Nature remains neutral with regard to jurisdictional claims in published maps and institutional affiliations. 